\title{
THE OPTIMAL CONTROL OF A JUMP MUTUAL INSURANCE PROCESS
}

\author{
By Charles S. Tapiero \\ Hebrew University, Jerusalem, Israel \\ University of Pennsylvania, Philadelphia
}

\section{INTRODUCTION}

We shall define a mutual insurance firm as a firm whose stockholders are the bearers of the insurance contracts issued by the firm. The firm's insurance is then viewed as a collective process of say $N$ persons seeking to protect themselves against claims that may occur to any one of them. For example, large employers protecting their employees by pooling risks and deducting for protection given amounts from salaries may be a case in point. In this latter case, the employer may match withdrawals from employees salaries and provide in the process a fringe benefit and increase employees loyalty to the firm. Alternatively, agricultural collectives have in some cases established mutual insurance firms whose purposes are to protect them, at a cost, from the uncertainty implicit in their production processes and the fluctuations of agricultural markets. Since these firms do not work for profit, contingent payments, or fund reimbursement in case of excess cash holdings are typical control policies which help cover extraordinary claims and at the same time are assumed the best investment policies. To further protect themselves against extraordinary claims, mutual insurers can turn to reinsurance firms, "selling" for example the excess claims of, say, a given amount $R$ (e.g., see TAPIERO and ZUCKERMAN (1982)). The purpose of this paper is to consider such a mutual insurance firm facing a jump stochastic claims process, as is often assumed in the insurance literature (e.g., FELLER (1971), BORCH (1974)). For example, Poisson and Compound Poisson processes are typical jump processes treated in this paper, although other processes could be considered as well (see SRINIVASAN (1973) and SRINIVASAN and Mehata (1976), Bensoussan and TAPIERo (1982)). First we define the mutual insurer problem as a jump process stochastic control problem which we solve analytically under the assumption of a gamma density claim sizes distribution. A solution is obtained by applying arguments from stochastic dynamic programming and by solving the resultant functional equation by application of Laplace transforms (e.g., Colombo and Lavoine (1972), Miller (1956), Tapiero, Chapter V). Subsequently, the effects of reinsurance are introduced and preliminary results obtained. As in TAPIERO and ZUCKERMAN (1982), we assume that the mutual insurance firm is a direct underwriter and that the reinsurer is a leader in a Stackleberg game (STACKLEBerg (1952), SimMAN and CruZ (1973), LuCE and RAIFFA (1957)). Although, in this latter case, analytical results are harder to obtain, numerical techniques can be applied to obtain practical results. Finally, it should be pointed out that this study, although applied to stationary parameters models to obtain analytical results, is equally valid for non-stationary parameters. 
In this vein, insurance problems under unstable environments and time varying processes can be investigated, leading to a more realistic appreciation of insurance firm's difficulties in the face of a dynamic environment.

\section{THE INSURANCE PROCESS}

Consider a mutual insurance firm with $N$ contracts at time $t$. Given $N$, the probability of a claim occurring in a small time interval $d t$, equals $\theta N d t$, where $\theta$ is a known parameter expressing the propensity of accidents' occurrences for each contract. Claim sizes are assumed to be randomly distributed with mean $m$, so that the mean claim in $d t$ equals $\theta N m d t$. Next, let the firm use a loading factor $\pi$ such that its average, deterministic cash income equals $(1+\pi) \theta \mathrm{Nm} d t$. At times $\tau_{i}, i=1,2, \ldots$ claims occur each with size $\xi_{i}, i=1,2, \xi_{i} \geqslant 0$, where as pointed out earlier $m=E\left(\xi_{i}\right)$ and with known distribution function $F(\cdot)$. If $x$ denotes the cash accumulation process over time, then

$$
\begin{aligned}
d x & =(1+\pi) \theta N m d t \\
x\left(\tau_{i}\right) & =x\left(\tau_{i}^{-}\right)-\xi_{i}, \quad i=1,2, \ldots \\
x(\theta) & =x_{0} .
\end{aligned}
$$

To characterize the stochastic process $\{x(t), t \geqslant 0\}$ formally, we define first the probability of jumps and jump occurrences. To do so denote a family of measures on $R^{n}$ by $M(x, t, d z)$ and assume that

$$
\begin{gathered}
\int_{R^{n}} M(x, t, d z) \leqslant C, \quad M(x, t, d z) \geqslant 0 \\
M(x, t,\{0\})=0 .
\end{gathered}
$$

Set

$$
M(x, t)=\int_{R_{n}} M(x, t, d z)
$$

and

$$
M^{1}(x, t, d z)=\frac{M(x, t, d z)}{M(x, t)} .
$$

Hence $M^{1}(x, t, d z)$ is a family of probability measures on $R^{n}-\{0\}$ and $M(x, t, d z)$ characterizes completely the jump process $\left(\tau_{i}, \xi_{i}\right), i=1,2, \ldots$ as follows.

$$
\operatorname{Prob}\left[\tau_{i+1} \geqslant s \mid \tau_{i}\right]=\exp \left[-\int_{\tau_{i} \Lambda s}^{s} M(x(u), u) d u\right]
$$

and

$$
\operatorname{Prob}\left[\xi_{i} \varepsilon A \mid \tau_{i}, x\left(\tau_{i}^{-}\right)\right]=\int_{A} M^{1}\left(x\left(\tau_{i}^{-}, \tau_{i}, d z\right)\right.
$$


where $\boldsymbol{A}$ is any set of $\boldsymbol{R}^{n}$. It is convenient to rewrite equation (1) as follows;

$$
x(t)=x(0)+\int_{0}^{t}(1+\pi) \theta N m d v-\int_{0}^{t} \int_{R^{n}} z \mu(d v, d z)
$$

where $\mu(t, A)$ is a function denoting the number of jumps of the process $x(t)$ in the time interval $(0, t]$ and $\mu(d v, d z)$ is a measure on $R^{+} \times R^{n}$ defined as follows; $(\Delta=(t, t+\Delta t))$;

$$
\mu(\Delta, A)=\mu(t+\Delta t, A)-\mu(t, A)
$$

and $A$ is a Borel subset on $R^{n}$. The measure $\mu(\Delta, A)$ is called the jump measure of the process $\{x(t), t \geqslant 0\}$. Next, assume that the firm incurs fixed administration costs proportional to the number of contracts $c N d t$ and that the firm can request from insurance contract holders contingent payments to meet extraordinary claims; in this case $u>0$ and $u$ is a controlled quantity. Alternatively, the firm can reimburse contract holders by distributing dividends, or plainly give back moneys (or reduce the premium payments equivalently) whenever cash levels reach the high levels. In this case $u<0$ and the firm cash-state equation (7) is reduced to

$$
x(t)=x(0)+\int_{0}^{t}[(1+\pi) \theta N m-c N+u] d t+\int_{0}^{t} \int_{R^{n}} z \mu(d v, d z)
$$

Evidently, $u$ may be of a feedback form $u(x)$, or be defined by an impulsive control structure, reflecting the fixed costs whenever extra-cash transactions (contingent payments or refunding) are incurred (e.g., see BENSOUSSAN and LIONS (1980), BENSOUSSAN and TAPIERO (1982)).

To demonstrate the type of probability processes considered here, assume as a special case that

$$
M(x, t, d x)=\delta(z-1) q(t)
$$

where $\delta(\cdot)$ is a Dirac-Delta function and $q(t)$ a known function. Then, from (3)

$$
M(x, t)=\int_{R^{n}} \delta(z-1) q(t)=q(t)
$$

and from (4)

$$
M^{1}(x, t, d z)=\frac{M(x, t, d z)}{M(x, t)}=\delta(z-1) .
$$

Hence from (5)

$$
\operatorname{Prob}\left[\tau_{i+1} \geqslant s \mid \tau_{i}\right]=\exp \left\{-\int_{\tau_{i} \Lambda s}^{s} q(v) d v\right\}
$$

also

$$
\mu(d v, d z)=\mu(d v) \delta(z-1)
$$


where $\mu(t)$ is a point process whose jump equals one or such that

$$
\begin{aligned}
& \operatorname{Prob}(\mu(d v)=1)=q d t+0(d t) \\
& \operatorname{Prob}(\mu(d v)=0)=1-q d t+0(d t) \\
& \operatorname{Prob}(\mu(d v) \geqslant 2)=0(d t) .
\end{aligned}
$$

Hence, $\mu(t)$ is a random variable distributed according to a Poisson law whose parameters is

$$
\Lambda(t)=\int_{0}^{t} q(v) d v .
$$

Of course, other point probability processes can be considered in this manner. Given the probability process (9), the firm's policies consist in selecting in some optimal manner the loading factor $\pi$ and the contingent payments (or dividend) $u$ of the firm. Next we define the firm's objective function which will subsequently be optimized. To obtain analytical (or numerical) results, it will be necessary to use some special assumptions regarding the firm objective function, which need not always reflect reality. Specifically, we assume linear costs consisting of cash carrying expenses $h x$, per unit time, a cost $g(\pi)$ associated to higher levels of loading factors, or $g(\pi)>0, \partial g / \partial \pi>0$. Throughout our optimization, $\pi$ will be assumed constant reflecting the firm's needs for constant income (from premiums). An additional cost associated to payments $u$ is given by $w|u|$, meaning that the firm seeks to avoid as much as possible extra contingent payments or refunding. Finally, a bankruptcy cost $K$ is incurred whenever the firm cash level reaches negative or zero states. At this time which we denote by $\tau$, the process terminates. In other words, the firm minimizes costs over a planning time $[0, \tau)$, where

$$
\tau=\operatorname{Inf}\{t>0 ; x \leqslant 0\}
$$

and for a given discount rate $r$, the firm objective is to:

$$
\underset{\pi, u}{\operatorname{Minimize}} J=E\left\{\int_{0}^{\tau}[h x+g(\pi)+w|u|] e^{-r t} d t+K e^{-r \tau}\right\} .
$$

Minimization of (11) subject to (9), (10) and a constraint on the size of possible extra payments (or refunding),

$$
|u|<U
$$

defines a stochastic control problem which we solve by dynamic programming. Throughout our solution we shall assume that the initial state $x(0)=x_{0}$ is given and that all parameters are constants, unless stated otherwise. These are simplifications made to obtain tractable results, although non-stationarity of parameters does not necessarily change the validity of our equations. 


\section{THE OPTIMAL CONTROLS: LINEAR COSTS}

Next we consider problem (11) without reinsurance and apply stochastic dynamic programming arguments to obtain for equation (11), the following integro-differential equation

$$
-r J(x)+q \int_{0}^{\infty}[J(x-\xi)-J(x)] f(\xi) d \xi-(\alpha+u) \frac{d J}{d x}+[h x+g(\pi)+w|u|]=0
$$

where $\alpha=(1+\pi) \theta N m-c N$. Minimization of (13) with respect to $u$, and (12), or

$$
\min _{u}\left\{-u \frac{d J}{d x}+w|u|\right\}
$$

leads to the following Bang-off-Bang control;

$$
u= \begin{cases}-U & \text { if } d J / d x<-w \\ 0 & \text { if }-w \leqslant d J / d x \leqslant w \\ +U & \text { if } w<d J / d x .\end{cases}
$$

This is found by noting that for $u>0$, we require for minimization that if $d J / d x<w$, then $u=0$ and if $d J / d x>w$ then $u=U$. By the same token, if $u<0$, then when $d J / d x<-w . u=-U$ minimizes (14) and when $d J / d x>-w, u=0$ is the minimum of (14). The Bang-off-Bang control given by (15) and Figure 1 below is of course practical as it allows a two modes policy of doing or not doing anything. The actual action chosen is a function of the marginal costs of $J(x)$, $d J / d x$, such that when the cost is too high we can reduce costs by increasing the income $(u=U)$ and when the original cost is too low we reduce the cost by reducing our cash holdings. Whenever $-w<d J / d x<w$, we do nothing. Inserting (15) into (13) we obtain an integro-differential equation which is given as follows (where subscripts are for convenience dropped).

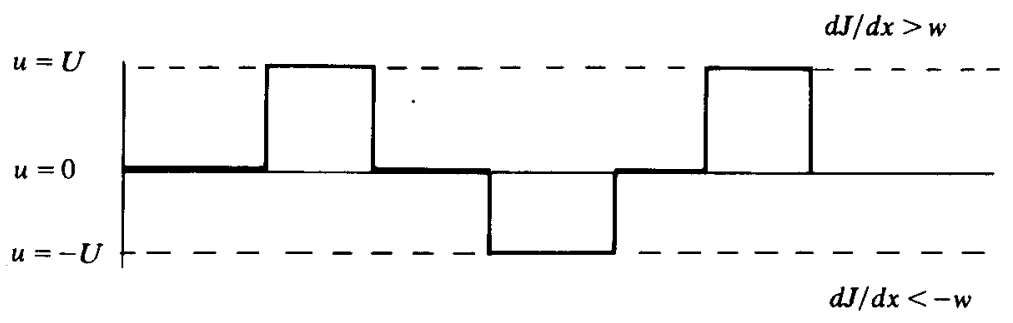

FIGURE 1. Bang-off-Bang control.

$$
-r J+q \int_{0}^{\infty}[J(x-\xi)-J(x)] f(\xi) d \xi+h x+g(\pi)+
$$


(16)

$$
\begin{cases}-(\alpha+U) \frac{d J}{d x}+w U=0 & \text { if } d J / d x>w \\ -\alpha \frac{d J}{d x}=0 & \text { if }-w<\frac{d J}{d x}<w \\ -(\alpha-U) \frac{d J}{d x}+w U=0 & \text { if } d J / d x<-w\end{cases}
$$

To resolve equation (16), we can proceed by an application of Laplace transforms LT (MILLER (1956)). Say that " $\mathscr{L}$ " denotes a LT operator where

$$
L(p)=\int_{0}^{\infty} e^{-p x} J(x) d x=\mathscr{L}\{J(x)\}
$$

Also

$$
\left\{\begin{array}{l}
\mathscr{L}\left\{\frac{d J}{d x}\right\}=p L(p)-J(0)=p L(p)-K \\
\mathscr{L}\left\{J(x-\xi)=e^{-\xi p} L(p) .\right.
\end{array}\right.
$$

Inserting into (16) where $u=(U, 0,-U)$, we have a $\mathrm{LT}$ of $J(x)$ given by

$$
L(p)=\frac{(\alpha+u) K+[g(\pi)+w|u|] / p+h / p^{2}}{(r+q)+(\alpha+u) p-q E\left(e^{-\xi p}\right)}
$$

where $u$ is, as pointed out earlier, a function taking on any one of three constant values $U$, zero and $-U$, and $E(\cdot)$ is an expectation operator. By the limit theorems for Laplace transforms,

$$
\left\{\begin{array}{l}
\lim _{x \rightarrow 0} J(x)=\lim _{p \rightarrow \infty} p L(p)=K \\
\text { and also } \\
\lim _{x \rightarrow \infty} J(x)=\lim _{p \rightarrow 0} p L(p)
\end{array}\right.
$$

we can obtain the boundary values for $J$. A solution for (18) can be expressed however if only we can give an explicit expression to $E(\exp -\xi p)$. For example, say that $\xi$ is a gamma probability distribution given by

$$
P(x)=\frac{\xi^{\gamma-1} e^{-(\xi / \beta)}}{\beta^{\gamma} \mu(\gamma)} ; \quad \gamma>0, \quad \beta>0, \quad \xi \geqslant 0
$$

then

$$
E\left(e^{-p \xi}\right)=(1+\beta p)^{-\gamma}
$$

which we insert into (18) to obtain

$$
L(p)=\frac{(1+\beta p)^{\gamma}\left[p^{2}(\alpha+u) K+p(g(\pi)+w|u|)+h\right]}{(1+\beta p)^{\gamma}\left[p^{2}(r+q)+p^{3}(\alpha+u)\right]-q p^{2}}
$$

for $\gamma$, an integer, the order of the denominator is $\gamma+3$ while that of the numerator equals $\gamma+2$. Thus, if we write $L(p)=Q_{1}(p) / Q_{2}(p)$, and solve for the $\gamma+3$ roots 
of $Q_{2}(p)=0, p_{1}, p_{2}, \ldots, p_{\gamma+1}, p_{\gamma+2}=P_{\gamma+3}=0$, then (18) can be written as follows:

$$
L(p)=\sum_{i=1}^{\gamma+1} A_{i} /\left(p-p_{i}\right)+A_{\gamma+2} / P+A_{\gamma+3} / P^{2}
$$

and the inverse transform of $L(p)$ is the sum of exponentials, or

$$
J(x)=\sum_{i=1}^{\gamma+1} A_{i} e^{p_{i} x}+A_{\gamma+2}+A_{\gamma+3} x
$$

also

$$
\frac{d J}{d x}=\sum_{i=1}^{\gamma+1} p_{i} A_{i} e^{p_{i} x}+A_{\gamma+3}
$$

Thus, a complete solution for $J$ is found since from (16),

$$
\begin{aligned}
J(x) & =k(x, U) & & \text { if } k^{\prime}(x, U)>w \\
& =k(w, 0) & & \text { if } k^{\prime}(x, 0)<w \\
& =k(x,-U) & & \text { if } k^{\prime}(x,-U)<-w
\end{aligned}
$$

where

$$
\begin{aligned}
& k(x, u)=\sum_{i=1}^{\gamma+1} A_{i}(u) e^{p_{i}(u) x}+A_{\gamma+2}(u)+A_{\gamma+3}(u) x \\
& k^{\prime}(x, u)=\sum_{i=1}^{\gamma+1} A_{i}(u) e^{p_{i}(u) x} p_{i}(u)+A_{\gamma+3}(u) .
\end{aligned}
$$

Thus, if we begin a $x=x_{0}$ at the initial state, we compute first in which mode $(u=0, U,-U)$ we are, according to (24) and then apply (25) to compute $J(x)$. Such computations are repeated for $J$ and $d J / d x$ as a function of $x$ and as soon as $d J / d x$ indicates a switching of mode, so is our computation of $J$ altered.

In the exponential claims case, $\gamma=1$ and equation (21) is given by;

$$
L()=\frac{h+p(\beta h+g(\pi)+w|u|)+p^{2}(K(\alpha+u)+\beta(g(\pi)+w|u|))+p^{3} \beta K(\alpha+u)}{2 p^{2}\left[r+p(\alpha+u+\beta(r+q))+p^{2}(\alpha+u)\right]} .
$$

The denominator in (26) has clearly a twice repeated root $p_{3}=p_{4}=0$ and two other roots given by

$$
p_{1,2}=-\frac{1}{2}[\alpha+u+\beta(r+q)] \pm \frac{1}{2}\left\{[\alpha+u+\beta(r+q)]^{2}-4(\alpha+u) r\right\}^{1 / 2}
$$

This means that $J(x)$ is of the form;

$$
J(x)=A, e^{p_{1} x}+A_{2} e^{p_{2} x}+A_{3}+A_{4} x .
$$

As a result, we can compute each of the parameters in (28) and provide a complete solution to the linear cost stochastic mutual insurance problem. 


\section{MUTUAL INSURANCE WITH REINSURANCE}

The mutual insurance process defined earlier can be extended further, and without difficulty, to include problems of reinsurance (see also TAPIERO and Zuckerman (1982), Dayananda (1970), Vajda (1955), Borch (1974)). Specifically, let $f(\xi)$ be the claims size distribution and let $R$ be a level above which the direct underwriter passes on the excess expense $\xi-R(\xi>R)$ to a reinsurer. As a result, the direct underwriter limits his actual claim expense to levels of at most $R$. To do so, the underwriter foregoes part of his income by paying a constant premium $y$, (as we shall see, function of $R$ the cut-off level) to the reinsurer. As a result, the direct underwriter's objective cost is given by (29) rather than (13);

$$
\begin{gathered}
-r J(x)+q \int_{0}^{R}[J(x-\xi)-J(x)] F(\xi) d \xi+q \int_{R}^{\infty}[J(x-R)-J(x)] f(\xi) d \xi \\
-(\alpha-y+u) \frac{d J}{d x}+[h x+g(\pi)+w|u|]=0
\end{gathered}
$$

and the objective cost $J(x)$, Laplace transform is (30) instead of (18);

$$
L(p)=\frac{[\alpha-y+u] K+[g(\pi)+w|u|] / p+h / p^{2}}{(r+q)+(\alpha-y+u) p-q\left[\int_{0}^{R} e^{-\xi p} f(\xi) d \xi+e^{-R p}(1-F(R))\right]}
$$

where $F(R)$ is the cumulative distribution of $f(\xi)$ from $\xi=0$ to $\xi=R$. Say that $f(\xi)=\mu \exp (-\mu \xi)$, then

$$
\int_{0}^{R} e^{-\xi p} f(\xi) d \xi+e^{-R p}(1-F(R))=\frac{\left[1-e^{-R(\mu+p)}\right]}{\mu+p}+e^{-R p}\left[1+\left[\frac{1-e^{-R \mu}}{\mu}\right]\right]
$$

which when introduced into (30) provides a Laplace transform for $J(x)$ still to be solved, either by approximation or by analytic inversion. Now assume for simplicity that the reinsurer seeks to maximize his expected cash holdings. We assume that $r=$ discount rate and $z=$ cash on hand, where

$$
\begin{array}{r}
d z=y d t \\
z\left(\tau_{i}\right)=z\left(\tau_{i}^{-}\right)-\rho, \quad \rho \geqslant 0 \text { and } \rho=\xi-R
\end{array}
$$

and the objective is;

$$
\underset{y}{\operatorname{Max}} \Phi(z)=E\left\{\int_{0}^{\tau} e^{-r t} z d t+Q e^{-r r}\right\}
$$

where $Q$ is the reinsurer bankruptcy cost. Without difficulty, it can be shown that $\phi$ is given by

$$
\begin{gathered}
-r \Phi(z)+q \int_{R}^{\infty}[\Phi(z+R-\xi)-\Phi(z)] f(\xi) d \xi-y \frac{d \Phi}{d z}-z=0 \\
\Phi(0)-Q
\end{gathered}
$$


which can be solved as pointed out earlier. The direct (mutual) underwriter and the reinsurer proceed then to solve the following game;

$$
\begin{aligned}
& \underset{\pi, u, R}{\operatorname{Min}} J(x \mid \pi, u, R, y) \\
& \underset{y}{\operatorname{Maximize}} \Phi(z \mid y) .
\end{aligned}
$$

Clearly, (34) defines a non-zero sum game. In this case, it is necessary to point out both the rules of the game, the informational assumptions available to each player and of course the market structure. For example, a leader-led market structure points out to a Stackelberg strategy (e.g., TAPIERO and ZUCKERMAN (1982), STACKelberg (1952), Simman and CRuz (1973)). Specifically, say that the reinsurer is a "leader" and dominates the game by imposing a solution which is favorable to itself. Then, for every $y$, the direct mutual underwriter minimizes the cost $J$ with respect to $\pi, u$ and $R$. This leads to solutions $\pi^{*}=\pi^{*}(y)$, $u^{*}=u^{*}(y)$ and $R^{*}=R(y)$. Insert the latter expression of $R(y)$ into (33) and then maximize $\Phi$ for $y$, or $y^{*}$. Then, the optimal direct mutual underwriter policies are given by $R^{* *}=R\left(y^{*}\right), u^{* *}=u^{*}\left(y^{*}\right)$ and $\pi^{* *}=\pi^{*}\left(y^{*}\right)$. Inversely, if the direct mutual underwriter is the leader then we first determine $y(R)$ which is introduced in the objective $J$ to yield optimal $\pi=\pi(y(R)), u=u(y(R))$ and $R=R(y(R))$.

\section{REFERENCES}

Bensoussan, A. and Lions, J. L. (1980). Controle impulsionnel et applications. Dunod: Paris.

BENSOUSSAN, A. and TAPIERO, C. S. (1982). Impulsive control: prospects and applications. Journal of Optimization Theory and Applications.

BORCH, K. (1974). The Mathematical Theory of Insurance. Lexington Books: Lexington, Mass.

Colombo, S. and LAvoire, J. (1972). Transformations de Laplace et de Mellin. Gautheir-Villars Editeur: Paris.

DayanANDA, P. A. W. (1970). Optimal reinsurance. Journal of Applied Probability 7, 134-156.

Feller, W. (1971). An Introduction to Probability and its Applications, Volume II. John Wiley \& Sons, Inc.: New York.

LUCE, R. D. and RAIFFA, H. (1957). Games and Decisions. John Wiley \& Sons, Inc.: New York.

Miller, S. K. (1956). Engineering Mathematics. Dover Publications: New York.

SimMAN, M., and CRUZ, J. B. (1973). Additional aspects of the Stackelberg strategy in non zero sum-games. Journal of Optimization Theory and Applications 11, 613-626.

Srinivasan, S. K. (1973). Stochastic Point Processes and their Applications. Griffin: London.

Srinivasan, S. K. and MehatA, K. M. (1976). Stochastic Processes. Tata McGraw Publishing Co. Ltd.: New Dehli.

Stackelberg, V. H. (1952). The Theory of Market Economy. Oxford University Press: Oxford.

TAPIERO, C. S. (1977). Managerial Planning: An Optimum and Stochastic Control Approach. Gordon Breach Science Publ.: New York.

TAPIERO, C. S. and ZUCKERMAN, D. (1982). Optimum excess-of-loss reinsurance: A dynamic framework. Stochastic Processes and Applications, 12, 85-96.

VAJdA, S. (ed.) (1955). Non-Proportional Reinsurance. E. J. Brill: Leyden. 


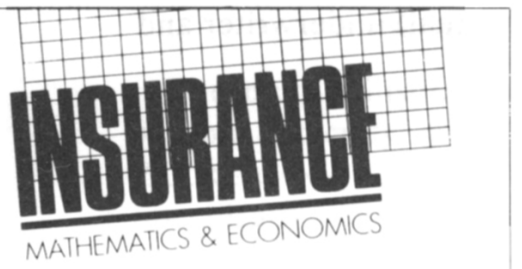

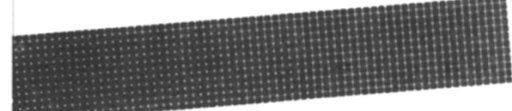

\section{Editors:}

F. De Vyider, Université Catholique de Louvain, Louvain-la-Neuve, Belgium;

H. Gerber, Université de Lausanne,

M. J. Goovaerts, Katholieke

Universiteit Leuven, Leuven, Belgium;

J. Haezendonck, Universiteit

Antwerpen, Wilrijk, Belgium;

S. Klugman, University of lowa,

lowa, U.S.A.;

G. C. Taylor, E.S. Knight and Co., Consulting Actuaries, Sydney, Australia
Switzerland;

\section{Aims and Scope}

INSURANCE: MATHEMATICS AND ECONOMICS is an international journal which intends to strengthen communication between individuals and groups who produce and apply research results in insurance mathematics, thus helping to correct the current fragmentation of research in the field. The journal feels a particular obligation to facilitate closer cooperation between those who carry out research in insurance mathematics and insurance economics (whether actuaries or non-actuaries) and practicing actuaries who are interested in the implementation of the results. To this purpose, INSURANCE: MATHEMATICS AND ECONOMICS will publish high quality papers of international interest, concerned with either the theory of insurance mathematics or the inventive application of it, including empirical or experimental results. Papers which combine several of these aspects are particularly welcome, as are survey papers of a pedagogical nature.

The subject matter of the Journal includes the theory, models and computational methods of life insurance (including pension systems, social insurance, and health insurance), of non-life insurance, and of reinsurance and other risk-sharing arrangements. It will also include, under the heading insurance economics, innovative insurance applications of results from other fields, such as probability and statistics, computer science and numerical analysis, economics, operations research and management science, and risk management.

\section{Major Topics Featured in this Journal}

Insurance Economics:

- Equilibrium Theory - Utility Theory - Portfolio Analysis

- Business Cycles - Inflation - Risk Management • Finance

- Accountancy - Investment - Profit-Sharing Arrangements

Mathematics Applied to Insurance:

- Ruin Theory • Graduation Theory - Claim Size and Claim Frequencies - Premium Principles - Classification of Risks - Experience Rating - Credibility Theory and Bonus-Malus Systems - Deductibles - Reserves - Risk-Sharing Arrangements - Reinsurance - Retentions - Catastrophic Risks

\section{Subscription Information}

The subscription price for Volume 1 in 4 issues (1982) is US $\$ 88.00 / \mathrm{Dfl}$. 220.00 including postage and handling.

The Dutch guilder price is definitive; US \$ prices are subject to exchange rate fluctuations.

\section{Specimen copies are available from the Publisher.}

\section{Associate Editors:}

J. A. Beekman, U.S.A.;

P. P. Boyle, Canada;

G. W. de Wit, The Netherlands;

N. Eshita, Japan;

R. Fortet, France;

E. Helten, W-Germany;

J. M. Hoem, Sweden;

W. S. Jewell, U.S.A.;

Y. Kahane, Israel;

J. J. McCutcheon, U.K.;

A. Mormino, Italy;

E. Neuburger, W-Germany;

R. Norberg, Norway;

E. Prieto Pérez, Spain;

A. F. Shapiro, U.S.A.;

J. Tougels, Belgium.
Please mail this coupon, or photo-copy to:

North-Holland Publishing Company,

P.O. Box 211, 1000 AE Amsterdam, The Netherlands.

Customers in the USA and Canada, mail to:

Elsevier Science Publishing Co., Inc.

52 Vanderbilt Avenue, New York, N.Y. 10017

Please send me a tree inspection copy of:

INSURANCE: MATHEMATICS \& ECONOMICS

Name:

Address: 